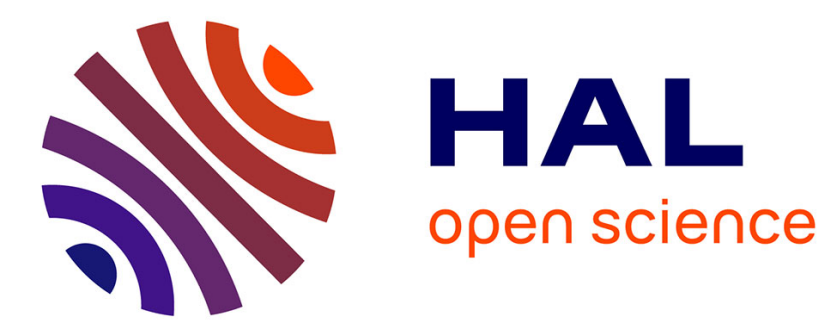

\title{
Surface Tension and Elasticity of Hexagonal Columnar Mesophases
}

\author{
L. Sallen, P. Oswald, J. Géminard, J. Malthête
}

\section{To cite this version:}

L. Sallen, P. Oswald, J. Géminard, J. Malthête. Surface Tension and Elasticity of Hexagonal Columnar Mesophases. Journal de Physique II, 1995, 5 (7), pp.937-961. 10.1051/jp2:1995222 . jpa-00248219

\section{HAL Id: jpa-00248219 https://hal.science/jpa-00248219}

Submitted on 1 Jan 1995

HAL is a multi-disciplinary open access archive for the deposit and dissemination of scientific research documents, whether they are published or not. The documents may come from teaching and research institutions in France or abroad, or from public or private research centers.
L'archive ouverte pluridisciplinaire HAL, est destinée au dépôt et à la diffusion de documents scientifiques de niveau recherche, publiés ou non, émanant des établissements d'enseignement et de recherche français ou étrangers, des laboratoires publics ou privés. 
Classification

Physics Abstracts

61.30-v - 61.30Jf $-62.20 \mathrm{Dc}$

\title{
Surface Tension and Elasticity of Hexagonal Columnar Mesophases
}

\author{
L. Sallen, P. Oswald, J.C. Géminard and J. Malthête \\ Laboratoire de Physique, Ecole Normale Supérieure de Lyon, 46 allée d'Italie, 69364 Lyon \\ Cedex 07, France
}

(Received 16 January 1995, received in final form 6 March 1995, accepted 5 April 1995)

\begin{abstract}
Résumé. - A l'aide de la méthode du joint de grain et en étudiant la forme d'équilibre d'inclusions liquides, nous avons mesuré la tension de surface et le diagramme de Wulff de l'interface hexagonal-isotrope du mélange lyotrope $\mathrm{C}_{12} \mathrm{EO}_{6}+$ eau et du cristal liquide discotique C8HET. Nous avons également obtenu la courbe d'énergie des parois symétriques dans les deux systèmes en fonction de l'angle de désorientation des colonnes. De l'analyse de cette courbe nous tirons le produit $K B$ des modules élastiques de courbure d'une colonne isolée et de compressibilité du réseau hexagonal. Ayant mesuré $B$ mécaniquement, nous en déduisons ensuite une estimation statique du module de courbure $K$ d'une colonne isolée. Nous trouvons dans les deux systèmes des valeurs de $K$ qui sont compatibles avec les données de rayons X.
\end{abstract}

\begin{abstract}
Using the grain boundary method and from the shape at equilibrium of liquid inclusions, we measured the surface tension and the Wulff plot of the hexagonal-isotropic interface of the lyotropic mixture $\mathrm{C}_{12} \mathrm{EO}_{6}+$ water and of the discotic liquid crystal C8HET. We also obtained the energy curve of the symmetric walls of the two systems as a function of the misfit angle of the columns. From their analysis we derive the product $K B$ of the elastic constants of curvature of an isolated column and of compressibility of the hexagonal array. Having measured $B$ mechanically, we then deduce a static estimate of $K$. In both systems, we find values of $K$ that are compatible with X-ray data.
\end{abstract}

\section{Introduction}

When put in presence of water, amphiphilic molecules, such as soaps, detergents or lipids, form aggregates of various shapes (plane, cylinder, sphere, minimal or random surfaces, etc). Usually, diluted phases are disordered at long range, whereas concentrated ones may form lyotropic liquid crystals which are translationnally ordered along one (lamellar phase), two (hexagonal phase) or three dimensions (cubic phase) [1].

The molecular structure of these mesophases is now well established. By contrast, their defects and their viscoelastic or interfacial properties are still poorly known. Also the hexagonal phase has been much less studied than the lamellar or the cubic phases. 


\section{$\mathrm{C}_{12} \mathrm{H}_{25}-\left(\mathrm{OCH}_{2} \mathrm{CH}_{2}\right)_{6}-\mathrm{OH}$}

\section{$\left(\mathrm{C}_{12} \mathrm{EO}_{6}\right)$}

a)

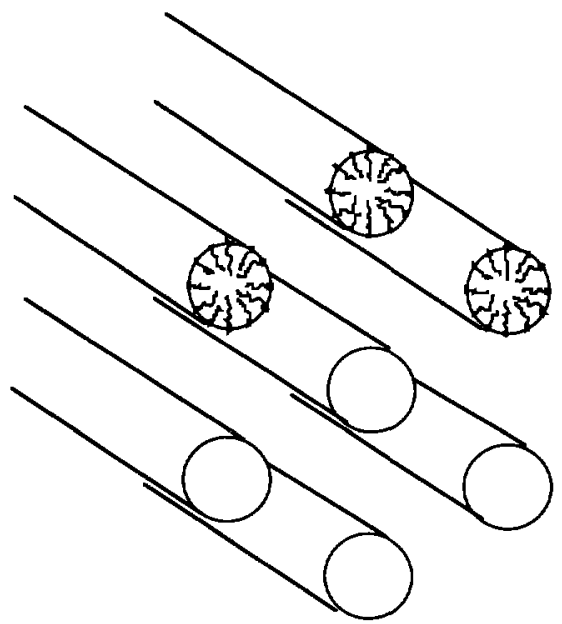

Direct hexagonal mesophase

b)

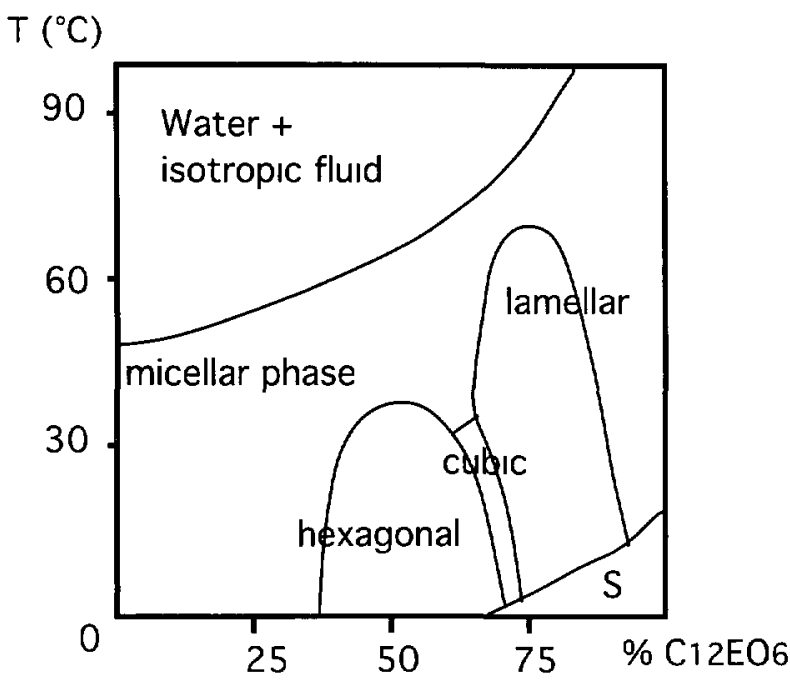

c)

Fig. 1 - a) $\mathrm{C}_{12} \mathrm{EO}_{6}$ molecule; b) Structure of a direct hexagonal mesophase; c) The phase diagram. 


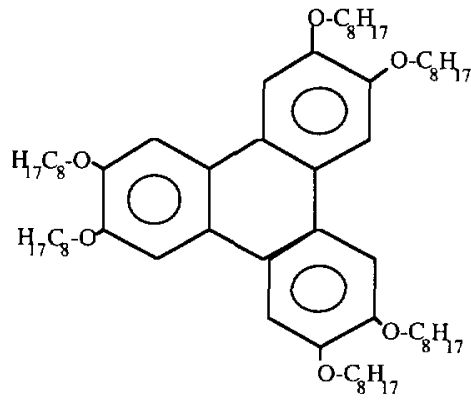

a)

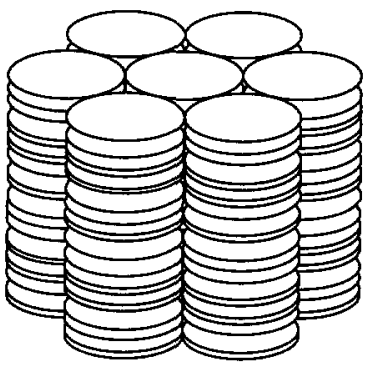

b)

Fig 2. - a) C8HET molecule; b) Columnar hexagonal mesophase.

For this reason, we have chosen to study the hexagonal phase of the binary mixture $\mathrm{C}_{12} \mathrm{EO}_{6}+$ water $[2,3]$ (Fig. 1). Another reason is that anomalies in the elastic coefficients have been reported in an equivalent thermotropic system. Indeed, Durand et al. $[4,5]$ found that the apparent curvature constant $K_{\text {app }}$ of the columns in the discotic liquid crystal C8HET (Fig. 2) is much larger than in nematics $\left(0.1\right.$ dyn rather than the expected $\left.10^{-6} \mathrm{dyn}\right)$. Their experimental method consisted in detecting the buckling threshold of the columns under mechanical stress [7], either in compression parallel to the columns [4]], or under dilation perpendicular to the columns [5]. Their results showed that these instabilities could still be explained in the theoretical framework of reference [7] (assuming a curvature-like elasticity of the columns) on condition that the curvature constant of the columns is increased by five or six orders of magnitude. The main conclusion of these experiments was that columns are correlated over a distance $\lambda_{\mathrm{app}}=\sqrt{K_{\mathrm{app}} / B} \approx 0.5 \mu \mathrm{m}\left(B \approx 10^{8} \mathrm{erg} / \mathrm{cm}^{3}\right.$ is the compressibility modulus of the hexagonal array) much larger than the lattice parameter (of the order of $30 \AA$ ). More recently, Durand et al. performed a Rayleigh scattering experiment in the same system and found that, when the columns are bent over distances larger than $0.3 \mu \mathrm{m}$, the columnar mesophase does not behave like a smectic $A$ but rather like a three-dimensional crystal [6a]. They concluded that they did not measure previously the curvature modulus of the columns (this is questionable) but rather a quantity (which is unfortunately not specified in [6a]) related to this solid-like elastic behavior. As pointed out by Durand in [6c] this does not mean that this columnar mesophase is a $3 \mathrm{D}$-crystal, which would be incompatible with $\mathrm{X}$-ray experiments, but only that there exists a mechanism that remains to be found which would lead to this apparent $3 D$-elastic behavior and to this very large value of $K_{\text {app }}$. A possible mechanism was proposed by Prost [8] who showed that a large density of column ends or entanglements could make the 
system stiffer and lead to an apparent curvature modulus $K_{\text {app }}$ much larger than $K$. But there is no experimental evidence of the existence of these defects so far.

In order to address this problem, we searched for a method to really measure the bending modulus $K$ of an ssolated column. It consists in observing grain boundaries and their intersections with the hexagonal-isotropic interface in a temperature gradient. Indeed, we shall see it is possible to derive an estimation of $K$ from a measurement of the energy of the grain boundaries as a function of the misfit angle of the columns. This method has the advantage of being purely static, in contrast to previous measurements using buckling instabilities.

In order to obtain the grain boundary energy, it was first necessary to determine the Wulff plot of the hexagonal-micellar interface. This is given in Section 2. We then used the grain boundary method to obtain the surface tension and the grain boundary energy simultaneously. This method consists in putting a thin sample into a temperature gradient and in looking at the grooves which form when grain boundaries intersect the hexagonal-micellar interface. By analyzing their shape as a function of the temperature gradient, it is possible to measure the surface tension and the corresponding grain boundary energy. These measurements are described in Section 3. In Section 4, we explain how to derive from these measurements the curvature constant $K$ of the columns and in Section 5, we give experimental results obtained by using the same method in the liquid crystal C8HET used by Durand et al. Finally, we compare our results to that obtained by Durand et al. in Section 4 .

\section{Wulff Plot}

In this experiment, we used a mixture whose concentration is very close to the azeotropic point (49.6\% in weight of polyoxyethylene). Samples are prepared between two parallel glass plates

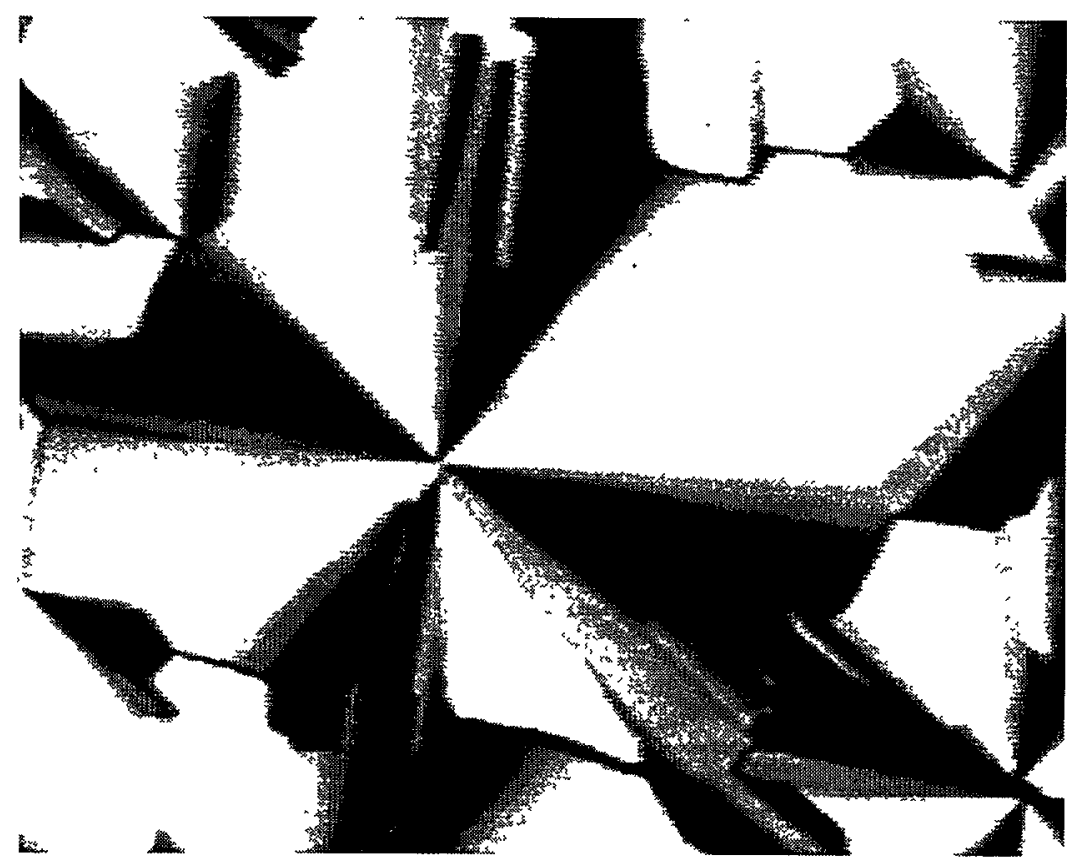

Fig. 3. - Fan-shaped structure. 


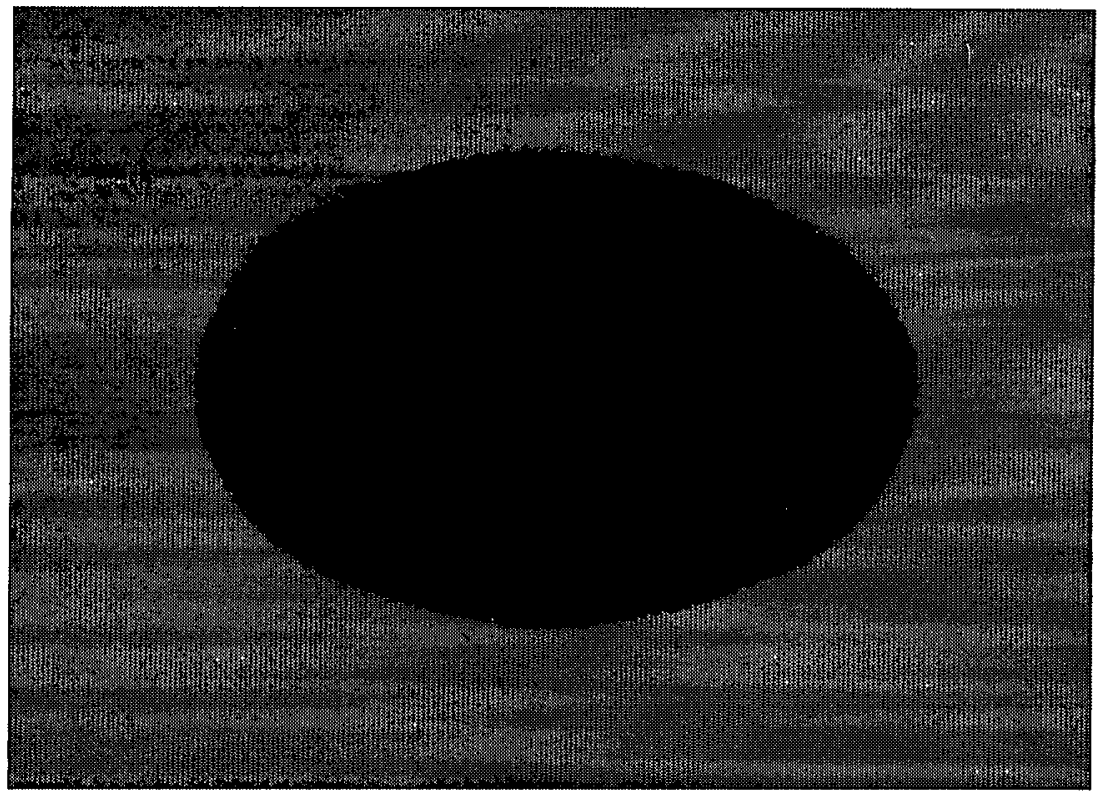

$50 \mu \mathbf{m}$

Fig. 4. - Liquid inclusion in equilibrium within a monodomain of hexagonal phase of $\mathrm{C}_{12} \mathrm{EO}_{6}$.

and are filled by capillarity in a moist atmosphere in order to avoid bubble formation and drying. They are then sealed with an epoxy glue (Epotecny Luxtrak LCR 0208). Our samples are homogeneous within $1 \%$ and can be kept at the transition temperature during many hours without visible drying and degradation. Figure 3 shows the fan-shaped texture which is usually obtained when a sample is homogeneously frozen from the isotropic micellar phase. In this texture, the molecular columns are parallel to the glass plates, but do not have a well defined direction. By contrast, directional solidification allowed us to make very good monodomains with all the columns oriented in a single direction (see Ref. [9]] for more details). Let us now describe how to obtain the Wulff plot in planar orientation.

Let $\gamma$ be the surface energy of the hexagonal-micellar interface. This quantity depends on the orientation of the interface with respect to the columns. The Wulff plot in planar orientation is, by definition, the polar plot $\gamma(\phi)$ where $\phi$ is the angle between the normal to the micellarhexagonal interface and a given crystallographic direction, for instance the average direction of the striations perpendicular to the columns. There are two possibilities to obtain this diagram: either by equilibrating a germ of the hexagonal phase in the micellar one or by doing the contrary. The first method is unusable because germs spontaneously disorient and grow by forming developable domains [10] as long as they are visible through the microscope. For this reason, we rather equilibrated an inclusion of isotropic liquid in a planar monodomain made in directional solidification. It is important to note that the columns keep their orientation when the hexagonal mesophase is partially melted and do not rearrange to form developable domains. This "locking" is certainly related to the apparent 3D-crystalline behaviour revealed by light scattering experiments [6a]. To reach equilibrium, we used an oven whose temperature is continuously adjusted in order to keep constant (within 1\%) the inclusion surface area. This experiment and the used experimental set up are described in detail in reference [11]. Of course the smaller the inclusion, the faster its equilibration time. Typically, one hour is necessary to 

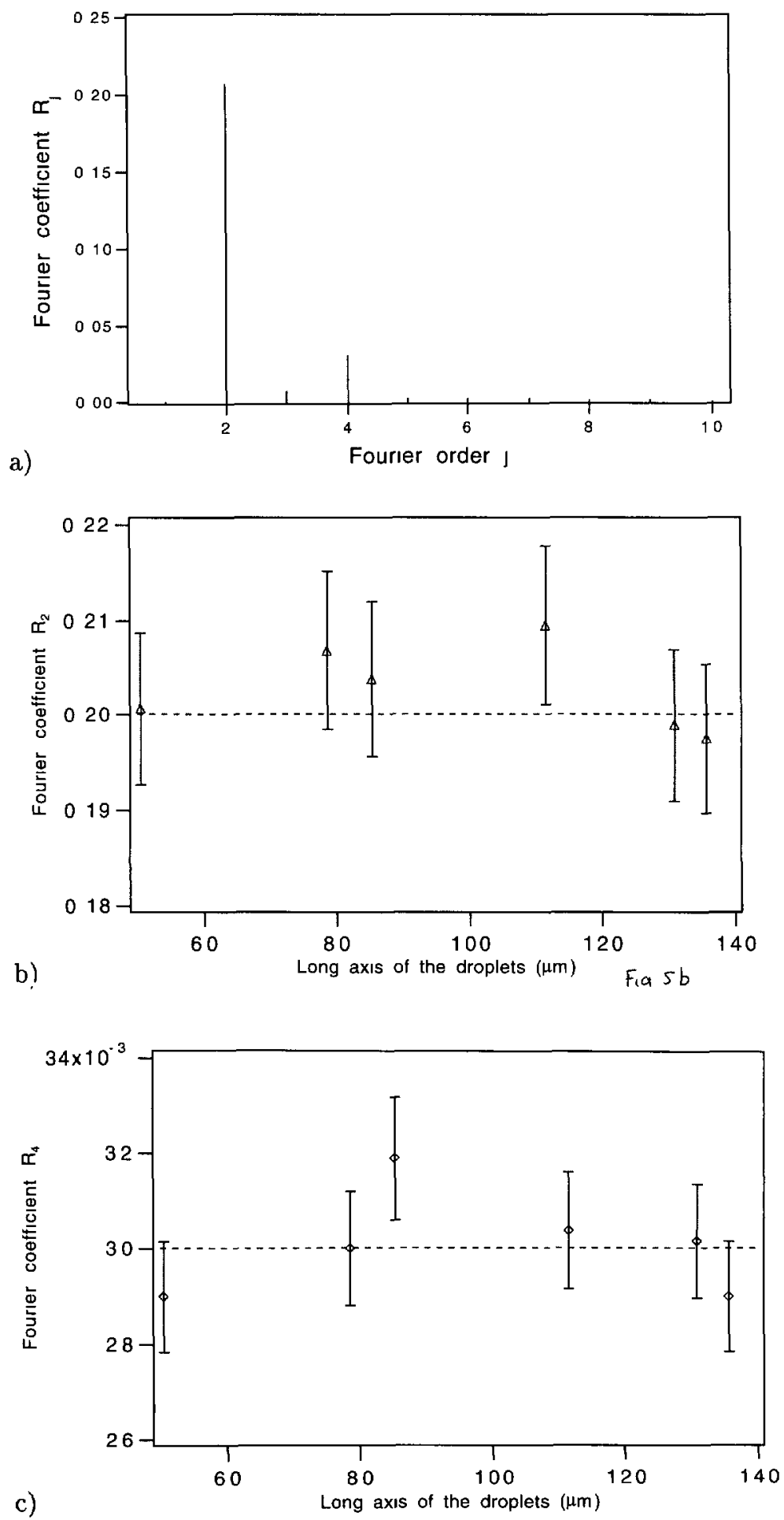

Fig. 5. - a) Fourier coefficient $R_{j}$ as a function of $j$; b) and c) $R_{2}\left(R_{4}\right)$ as a function of the long axis of the liquid inclusion 


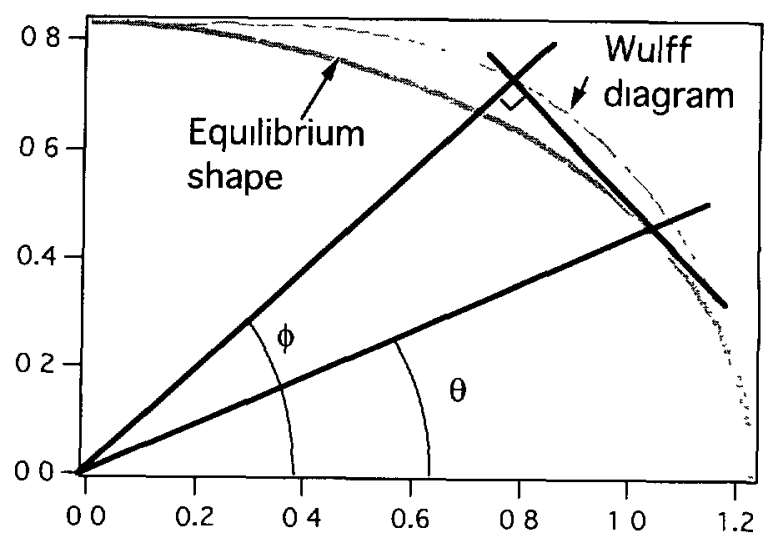

Fig 6. - Wulff's construction.

equilibrate an inclusion of diameter $50 \mu \mathrm{m}$ (Fig. 4). To be sure that we reached equilibrium, we performed the same experiment many times by starting from inclusions of various shapes and dimensions. Each time, we checked that the final shape was similar to others. Let $R(\theta)$ be the polar equation of a germ. Because of the two-fold symmetry, one must have at equilibrium:

$$
R(\theta)=R_{0}\left[1+R_{2} \cos (2 \theta)+R_{4} \cos (4 \theta)+\ldots\right]
$$

where $R_{2}, R_{4}, \ldots$ are the two-fold, four-fold,... anisotropies. In Figure 5, we plotted them as a function of the size of the inclusion. These quantities are constant within $5 \%: R_{2} \approx 0.2, R_{4} \approx$ 0.03 and do not depend on the sample thickness which we varied from $20 \mu \mathrm{m}$ to $100 \mu \mathrm{m}$. This result is important and shows that meniscus effects in the sample thickness are negligible [12]. Using the Wulff construction [13], it is now possible to obtain the Wulff plot $\gamma(\phi)$ (Fig. 6) and to show that

$$
\gamma(\phi)=\gamma_{0}\left[1+\gamma_{2} \cos (2 \phi)+\gamma_{4} \cos (4 \phi)+\ldots\right]
$$

with $\gamma_{2}=0.194$ and $\gamma_{4}=-0.013$.

In this expression, $\phi$ is the angle between the tangent to the interface and the molecular columns.

Equation (2) shows that the surface energy is minimal when the columns are perpendicular to the interface. This means that ends of columns are energetically favourable at the hexagonalmicellar interface. Let us now discuss how to obtain the prefactor $\gamma_{0}$.

\section{The Grain Boundary Method}

This is one of the most general methods used by metallurgists for measuring the solid-liquid surface energy [14]. It consists in observing the shape of the hexagonal-micellar interface constrained by the presence of a grain boundary between two disoriented domains.

In order to obtain a flat hexagonal-micellar interface, we put a planar sample in a linear temperature gradient $G$. When a grain boundary (or a wall) separating two developable domains of the fan texture intersects the interface, a groove is formed (Fig. 7a): its shape and its depth obviously depend on the wall energy, on the surface tension and its anisotropy and 


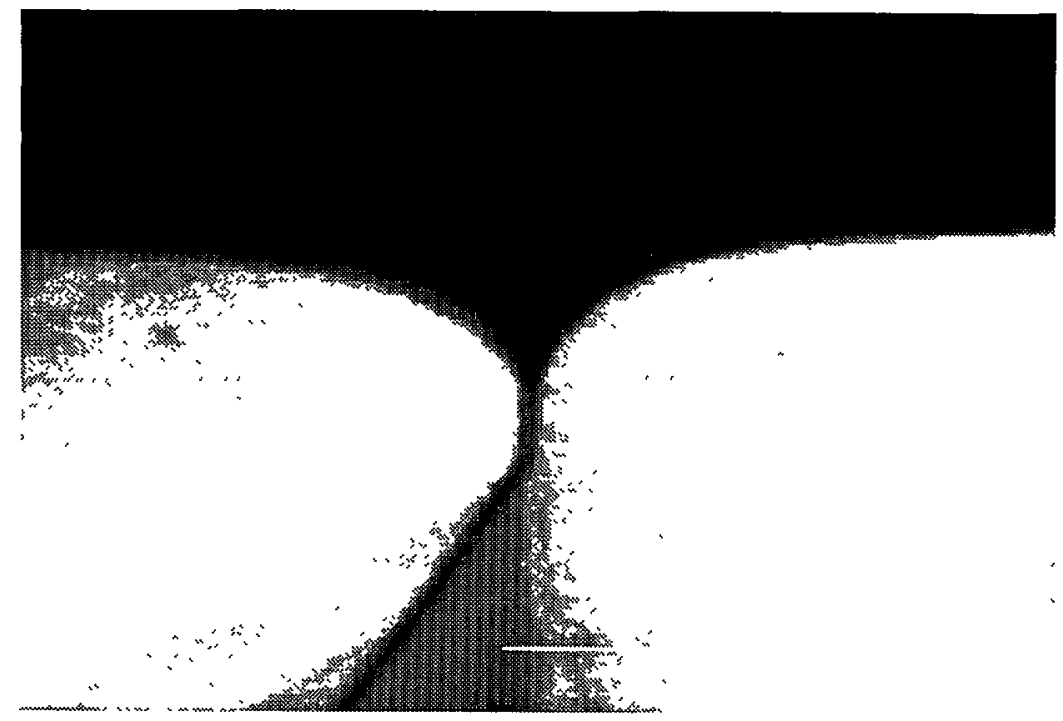

a)

Isotropic fluid

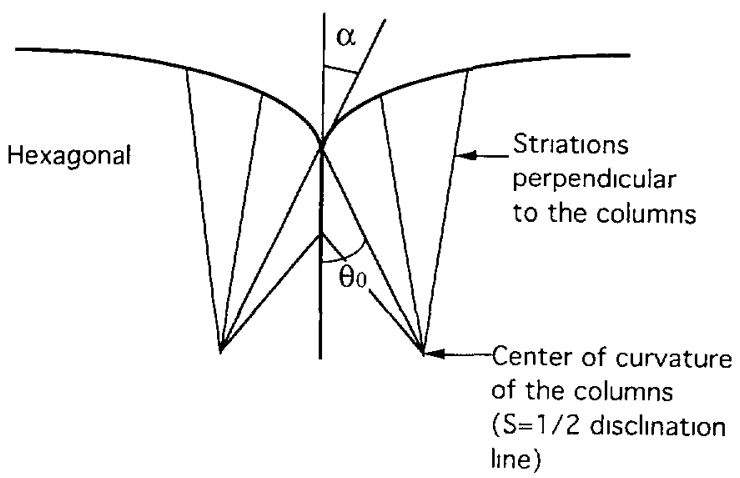

b)

Fig. 7. - a) Groove formed at the intersection of a grain-boundary with the isotropic- hexagonal interface $\left(G=24{ }^{\circ} \mathrm{C} / \mathrm{cm}\right)$; b) Definition of angles $\alpha$ and $\theta_{0}$.

on the temperature gradient. To simplify, we shall only analyze symmetric walls of the first species $[10,15]$ (Fig. 7b). Let $2 \theta_{0}$ be the misfit angle of the columns at point $\mathrm{P}$ (at the bottom of the groove), $E\left(\theta_{0}\right)$ the wall energy and $\alpha$ the angle between the tangent to the interface and the wall at this point. The equilibrium condition of point $\mathrm{P}$ is [16].

$$
E\left(\theta_{0}\right)=2\left[\gamma(\phi) \cos (\alpha)-\gamma^{\prime}(\phi) \sin (\alpha)\right]
$$

where $\gamma^{\prime}=\mathrm{d} \gamma / \mathrm{d} \phi$. This equation gives the wall energy, provided $\gamma(\phi)$ and are known. The shape and the depth $h$ of the groove are given by integrating the Gibbs-Thomson relation. If 


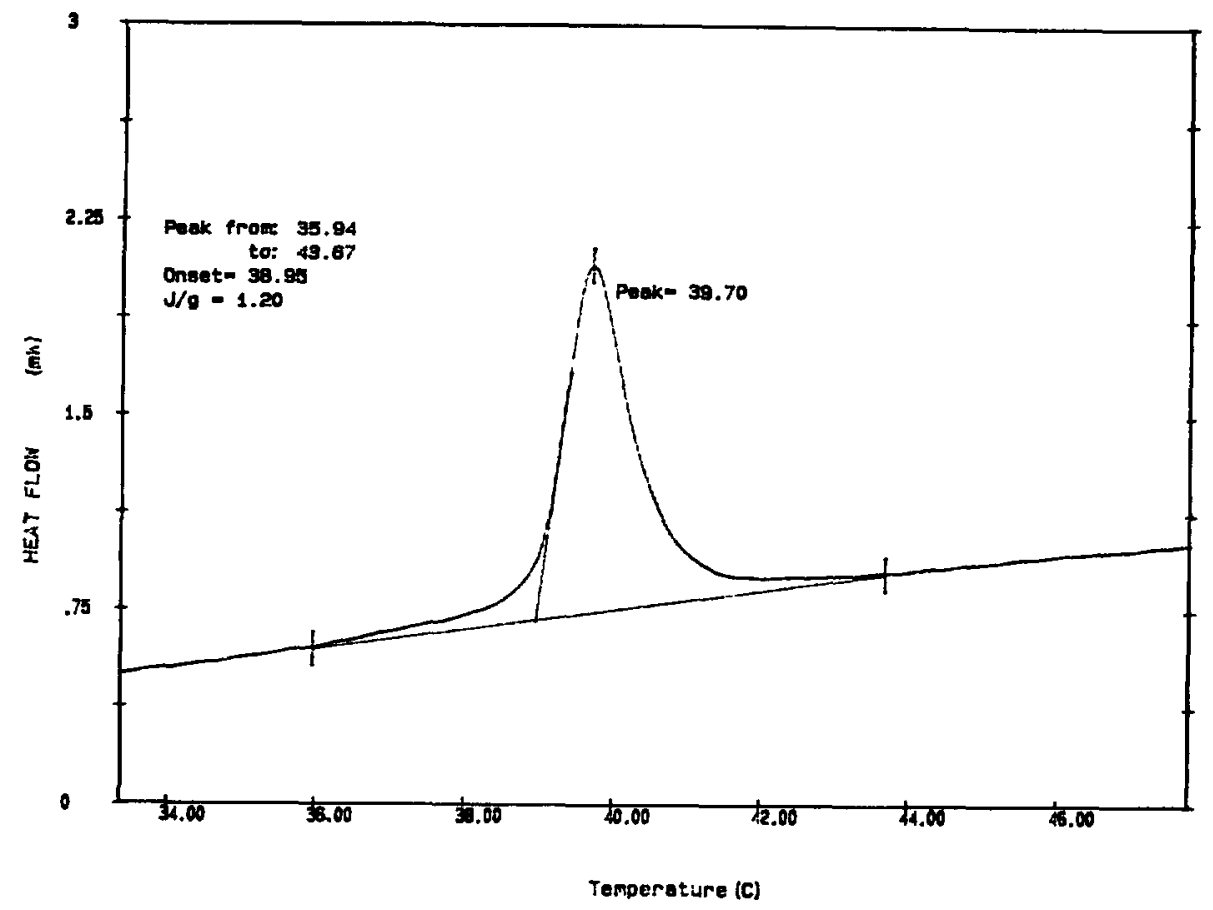

Fig. 8. - Heat flux versus temperature (scannıng velocity is $5^{\circ} \mathrm{C} / \mathrm{mn}$ ).

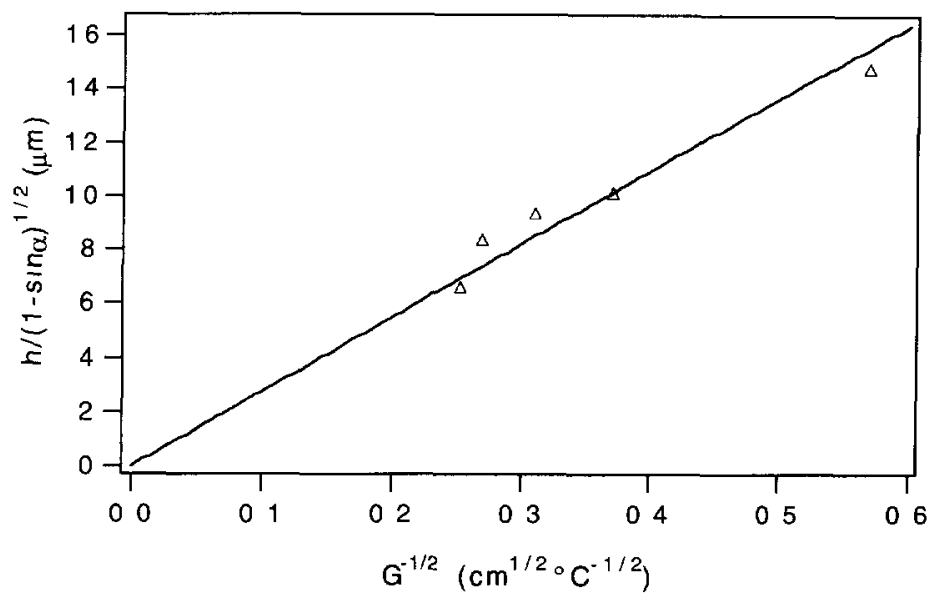

Fig. 9. - Groove depth $h$ as a function of temperature gradient $G$.

the surface energy is isotropic $\left(\gamma(\phi)=\gamma_{0}\right)$, this calculation can be done analytically and yields:

$$
h=\sqrt{\frac{2 \gamma_{0}(1-\sin \alpha) T_{0}}{G L}}
$$

where $T_{0}$ is the melting temperature and $L$ the latent heat per unit volume that we found to 

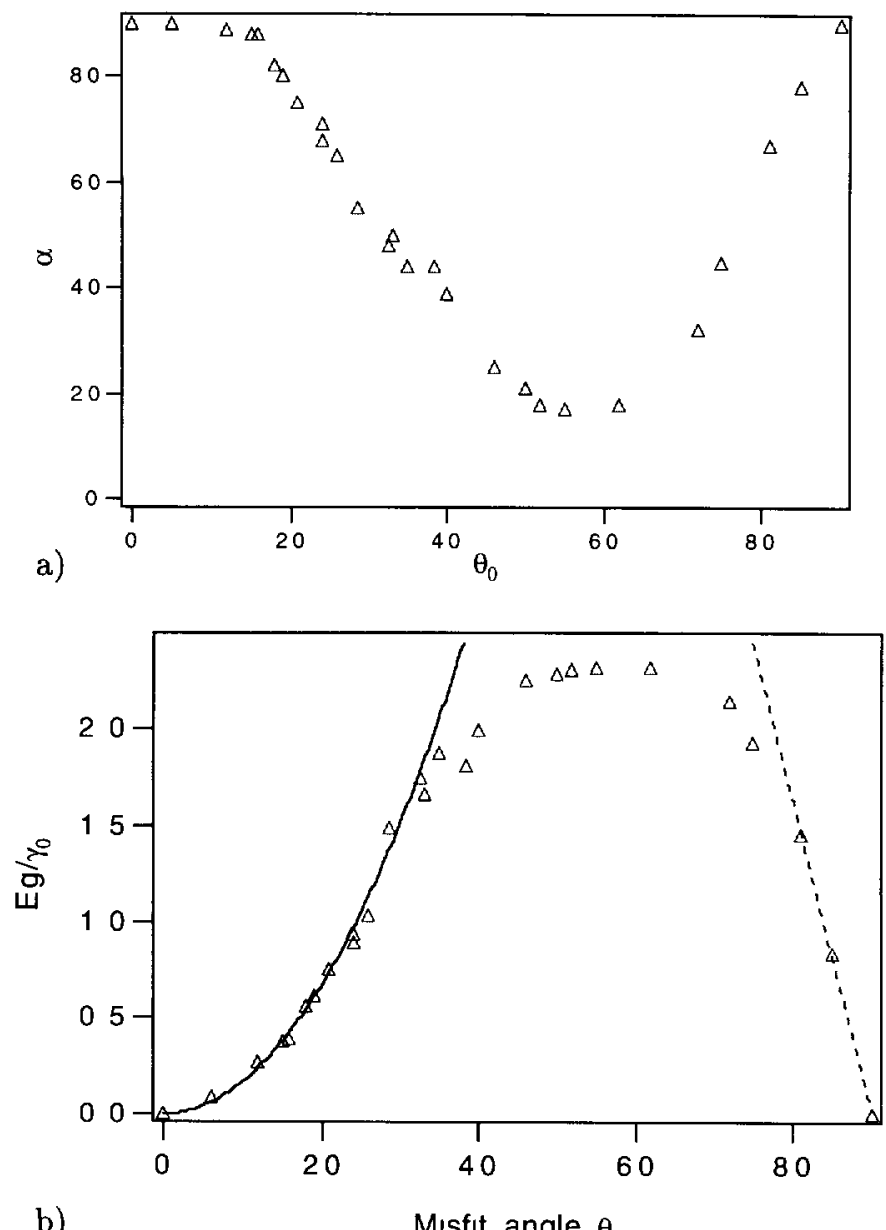

b)

Misfit angle $\theta_{0}$

Fig. 10. - a) Angle $\alpha$ as a function of angle $\theta_{0}$; b) Grain-boundary energy (in $\gamma_{0}$ unit) versus $\theta_{0}$ $\left(\mathrm{C}_{12} \mathrm{EO}_{6}+\right.$ water mixture $)$.

be close to $1.2 \times 10^{7} \mathrm{erg} / \mathrm{cm}^{3}$ in our system (Fig. 8). This value is in good agreement with previous measurements of Clunie et al. [17]. Equation (4) shows that $h / \sqrt{1-\sin \alpha}$ must vary like $G^{-1 / 2}$. We checked this dependence experimentally (Fig. 9) and found $\gamma_{0} \approx 0.22 \mathrm{erg} / \mathrm{cm}^{2}$ with the help of equation (4).

This value is approximate because we have neglected the surface tension anisotropy (about $20 \%$ in our system). For this reason we used another method proposed by Arbel and Cahn [18] to go through our experimental data (see Appendix A). This method allowed us to take into account surface tension anisotropy given in the preceding section. This gives:

$$
\gamma_{0} \approx 0.26 \pm 0,02 \mathrm{erg} / \mathrm{cm}^{2}
$$

We shall use this value in the following.

We are now in a position to determine the wall energy as a function of the misfit angle $\theta_{0}$ using equation (3) Indeed $\alpha$ and $\theta_{0}$ can be easily measured through the polarizing microscope. 
Our experimental results are given in Figure 10.

\section{Determination of the Curvature Constant $\mathrm{K}$}

We know from previous studies [15] that different types of wall exist in hexagonal mesophases depending on the value of the misfit angle $\theta_{0}$.

If $\theta_{0}$ is very small (Fig. 11a), columns bend continuously and form a wall of curvature of energy

$$
E_{\mathrm{c}} \approx \frac{2}{3} \sqrt{K B} \theta_{0}^{3}
$$

where $K$ is the curvature constant of the columns and $B$ the compressibility modulus of the hexagonal array.

This energy quickly increases with angle $\theta_{0}$ because of the dilation of the hexagonal array in the midplane of the wall. A convenient way to reduce this dilation is to introduce pairs of transverse edge dislocations at regular distances $l$ (Fig. 11b), a model that has already been proposed in smectics by Williams [19]. The best compromise is obtained when $l=2 b / \theta_{0}^{2}$ where $b$ is the Burger vector of the dislocations: in this case, the dilation of the hexagonal array is suppressed in the plane of symmetry of the wall. The wall energy is thus essentially due to dislocations:

$$
E_{\mathrm{cd}} \approx \frac{\theta_{0}^{2}}{2 b} 2 E_{\mathrm{d} \mathrm{sl}}
$$

The subscript "cd" means that we are dealing with a curvature wall containing dislocations and $E_{\mathrm{d} 1 \mathrm{sl}}$ is the energy of a tranverse edge dislocation that we know to be equal to $[7,20]$ :

$$
E_{\mathrm{d} \mathbf{s} \mathrm{l}}=\sqrt{\frac{K B b^{2}}{8 r_{\mathrm{c}}}}+2 r_{\mathrm{c}} \gamma_{0}
$$

The first term represents the elastic contribution to energy while the second is a core energy (we assume that the core is dissociated in its glide plane and has energy $\gamma_{0}$ per unit surface). Minımization as a function of the core radius $r_{\mathrm{c}}$ gives

$$
E_{\mathrm{d} 1 s \mathrm{l}} \approx(K B)^{1 / 4}\left(\gamma_{0}\right)^{1 / 2} b
$$

and

$$
E_{\mathrm{cd}} \approx(K B)^{1 / 4}\left(\gamma_{0}\right)^{1 / 2} \theta_{0}^{2}
$$

This dependence in $\theta_{0}^{2}$ shows that this combined wall is rapidly better than a curvature wall when $\theta_{0}$ increases (in fact when $\theta_{0}>10^{\circ}$ ). For this reason we used equation (10) to fit our experimental data in the range $10<\theta_{0}<30^{\circ}$. The best fit (solid line in Fig. 10b) gives

$$
\frac{\sqrt{K B}}{\gamma_{0}} \approx 31
$$

This value must be regarded as an order of magnitude. Indeed, we only took into account the self energy of dislocations, neglecting elastic interactions between them. Nevertheless, this approximation seems to be reasonable when dislocations are far enough from each other. This is the case when $\theta_{0}$ is small. Let $l$ be the distance between two dislocations in the same glide 


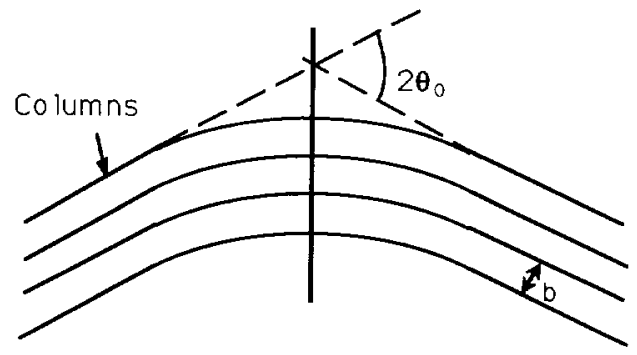

a)

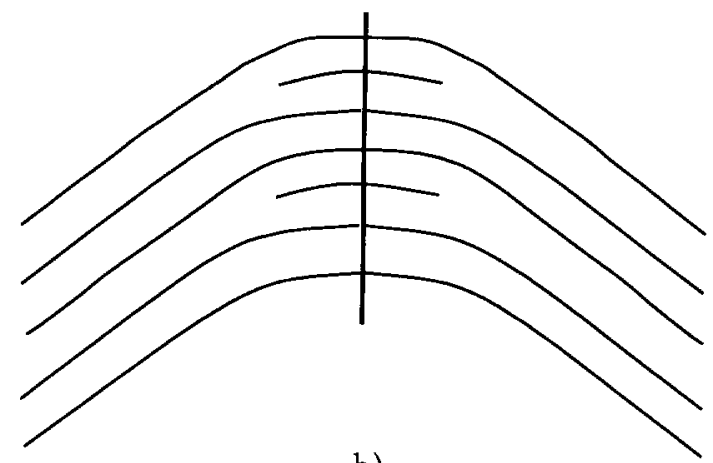

b)

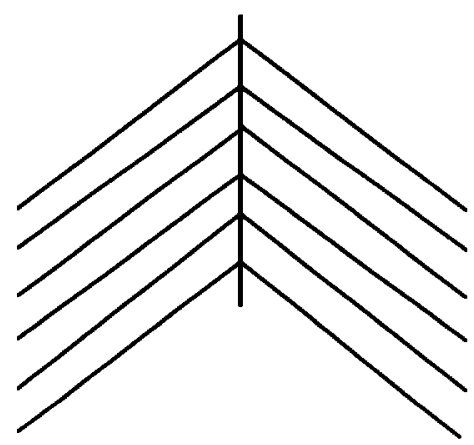

c)

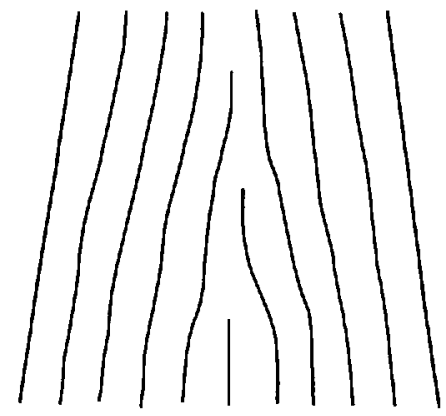

d)

Fig. 11. - a) Curvature wall; b) Mixed wall; c) Discontinuity wall; d) Sub-grain boundary. 
plane (Fig. 11b); for $\theta_{0}=10^{\circ}, l=2 b / \theta_{0}^{2} \approx 0.25 \mu \mathrm{m}$ (with $b=40 \AA$ ). According to Williams and Kléman's calculations [21], their interaction energy reads:

$$
W=\frac{1}{4} B b^{2}\left(\frac{\lambda}{\pi l}\right)^{1 / 2}
$$

where $\lambda=(K / B)^{1 / 2}$ is the penetration length. As $E_{\mathrm{d}_{1 \mathbf{s}}} \approx B b^{2}$ (because $r_{\mathrm{c}} \approx \lambda$ ) we calculate $W / E_{\mathrm{disl}} \approx \sqrt{\lambda / \pi l} \approx 2 \%$ by taking $\lambda=6 \AA$ (see hereafter) and $l=1 \mu \mathrm{m}$. In fact, this ratio is always small in our experiment (it equals $6 \%$ when $\theta_{0}=30^{\circ}$ ), which justifies our approximation a posteriori .

When $\theta_{0}$ ranges from $40^{\circ}$ to $70^{\circ}$, the density of dislocations becomes very large and it is more reasonable to suppose that the wall "melts" locally ("wall of discontinuity", Fig. 11c) [14]. This interpretation is sustained by measurements since we have in this regime (Fig. 10b):

$$
E_{\mathrm{d}} \approx 2 \gamma_{0}
$$

Finally, the energy decreases when $\theta_{0}>70^{\circ}$ to vanish when $\theta_{0}=90^{\circ}$ since the columns are again parallel to each other. When $\theta_{0}$ is close to $90^{\circ}$, the wall is composed of dislocations separated from each other by distance $\left(\pi-2 \theta_{0}\right) / b$ (Fig. 11d). In this limit, the wall energy reads:

$$
E_{\mathrm{sgb}} \approx(K B)^{1 / 4}\left(\gamma_{0}\right)^{1 / 2}\left(\pi-2 \theta_{0}\right)
$$

As previously we only sum the self energies of dislocations. This procedure is justified because the elastic interaction between dislocations vanishes when they are in the same climb plane [21]. The best fit to the experimental data (dashed line in Fig. 10b) gives:

$$
\frac{\sqrt{K B}}{\gamma_{0}} \approx 23
$$

in satisfactory agreement with our previous estimation. In the following we shall take $\frac{\sqrt{K B}}{\gamma_{0}} \approx$ $27 \pm 4$. Using $\gamma_{0} \approx 0.26 \pm 0,02 \mathrm{erg} / \mathrm{cm}^{3}$, we obtain

$$
\sqrt{K B} \approx 7 \mathrm{erg} / \mathrm{cm}^{2}
$$

As a conclusion, the grain boundary method allowed us to estimate the product $K B$. To measure $B$, we used a special cell equipped with piezoelectric ceramics [22]. This cell and the $B$ measurement are described in Appendix B. We found

$$
B \approx 1.2 \times 10^{8} \mathrm{erg} / \mathrm{cm}^{3}
$$

at the transition temperature, which gives

$$
\lambda \approx 6 \AA \text { and } K \approx 4 \times 10^{-7} \text { dyn. }
$$

This value of $K$ is of the same order of magnitude as in usual nematics. The value of $\lambda$ is also very close to that found by $\mathrm{X}$-ray diffraction by Clerc in this system at another temperature and concentration ( $\lambda \approx 20 \AA$ at $T=20{ }^{\circ} \mathrm{C}$ for a mixture with $55 \%$ in weight of $\mathrm{C}_{12} \mathrm{EO}_{6}$ ). We also performed the same experiment in the discotic liquid crystal. Our results are summed up in the next section. 


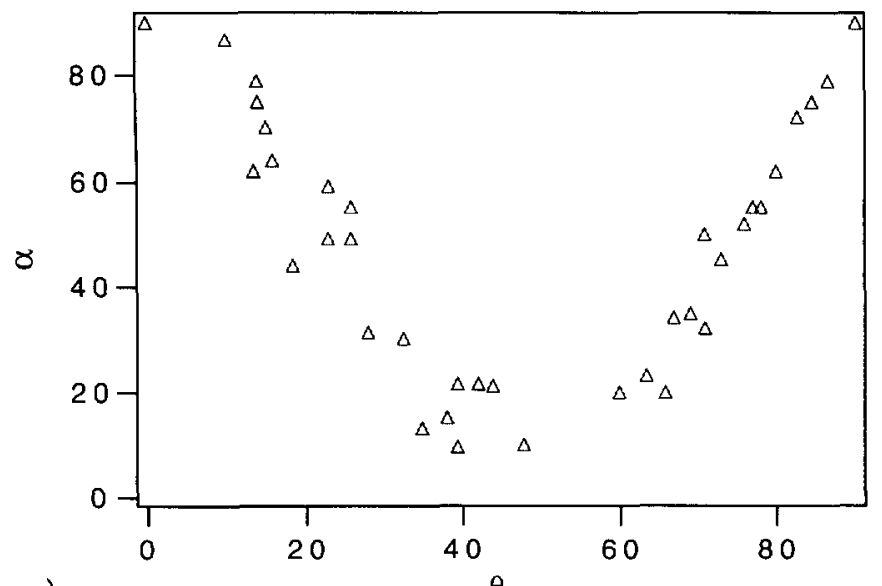

a)

$\theta_{0}$

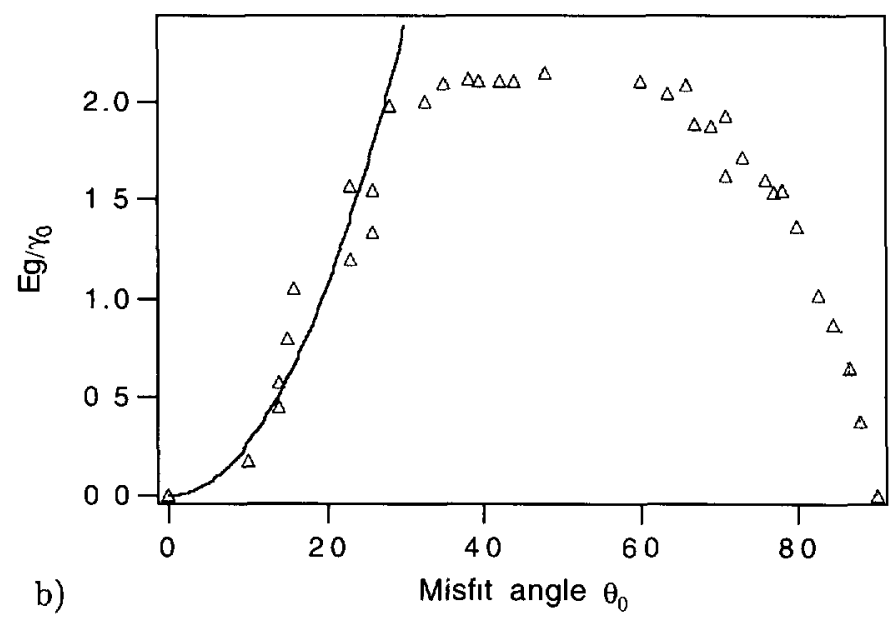

Fig. 12. - a) Angle $\alpha$ as a function of angle $\theta_{0}$; b) Grain-boundary energy (in $\gamma_{0}$ unit) versus $\theta_{0}$ (C8HET liquid crystal).

\section{Comparison with the Discotic Liquid Crystal}

In order to know whether our value of $K$ is specific to lyotropics, we performed the same experiments in C8HET.

The main difficulty was to prepare planar samples. Indeed, unlike lyotropics, it is much easier without surface treatment to obtain homeotropic orientation in thin samples of discotics rather than planar one. To force planar aligment, we coated our glass plates with a silane (ZLI 3124 from Merck Corp.) which is known to give homeotropic anchoring with nematics. In this case, disc-like molecules prefer to be perpendicular to the glass and planar alignment of the columns is slightly favored. In addition, directional solidification at very small velocity (less than $1 \mu \mathrm{m} / \mathrm{s}$ ) is necessary to obtain good planar domains and symmetrical walls. Indeed, homeotropic anchoring is always observed at large pulling velocity whatever the surface treatment. Our experiments being similar to the previous ones, we just report our experimental findings in Figure 12. This curve (which was obtained by taking $\gamma_{2} \approx 0.117$ [23]) closely 
resembles that obtained with lyotropics. The best fit with theoretical laws (10) and (14) gives

$$
\frac{\sqrt{K B}}{\gamma_{0}} \approx 50
$$

This ratio is larger than that found in lyotropics. As for $\gamma_{0}$ and $B$, they have been measured previously [24] and are respectively equal to $0.52 \mathrm{erg} / \mathrm{cm}^{2}$ and $10^{8} \mathrm{erg} / \mathrm{cm}^{3}$. With these values, we calculate:

$$
\lambda \approx 26 \AA \text { and } K \approx 6.8 \times 10^{-6} \mathrm{dyn}
$$

This value is about 10 times larger than in discotic nematics [25] and 30 times larger than the value obtained by X-ray diffraction by Davidson et al. [26] in the hexagonal phase of a similar system (hexahexylthiotriphenylene): $K \approx 1-4 \times 10^{-7}$ dyn.

\section{Concluding Remarks}

Our main result is that the value of the curvature constant $K$ is, as expected, of the same order of magnitude as in nematics or smectics. This value has been obtained by measuring the energy of edge dislocations. Because the column distorsions are mainly localized within a small region around the core of dislocations, this measurement only reflects the elastic behavior of the phase at a micrometric scale. The smallness of $K$ also explains why a germ of the hexagonal phase in equilibrium with its isotropic liquid disorients spontaneously by forming developable domains when it is extremely small, i.e., of a few $\mathrm{mm}$ in diameter. This result shows again that the hexagonal phase behaves as a usual liquid crystal at a micrometric scale. This phenomenon is equivalent to the formation of "Friedel bâtonnets" in smectics and comes from a competition between surface tension anisotropy $\Delta \gamma \approx \gamma_{0} \gamma_{2}$ and curvature elasticity $K$ [27]. It occurs when the germ size is larger than roughly $K / \Delta \gamma$. In our systems, this critical size is of the order of $1 \mu \mathrm{m}$ in discotics and is still smaller in lyotropics, which agrees qualitatively with observations.

Let us see now whether we can use our values of $K$ at a large scale, for instance when calculating the core radius and the energy of a developable domain of a macroscopic size (disclination). We know from previous observations $[15,28]$ that the core of such a line defect is very large (a few tenths of a $\mu \mathrm{m}$ in diameter) in discotic systems. By contrast, in lyotropics, the core size is too small to be measurable with an optical microscope. A standard calculation, assuming a curvature-like elasticity of the columns at large scale, gives for an $S=1$ disclination:

$$
E_{\mathrm{dev}}=\pi K \ln \frac{R}{r_{\mathrm{c}}}+E_{\mathrm{c}}
$$

where $R$ is the outer radius of the domain (100 $\mu \mathrm{m}$ typically in usual textures), $r_{\mathrm{c}}$ the core radius of the disclination and $E_{\mathrm{c}}$ its core energy. In this calculation we have assumed for simplicity that the cylinders are circle-shaped and centered on the disclination axis. To calculate the core radius we can imagine that either the core is filled with hexagonal mesophase, or it is filled with isotropic liquid.

The first model was already proposed in reference [15]. In this model, the columns in the core are parallel to the disclination axis and the core energy reduces to a surface term of the order of

$$
E_{\mathrm{c}} \approx 2 \gamma_{0} 2 \pi r_{\mathrm{c}}
$$

where $2 \gamma_{0}$ is the measured energy for a discontinuity wall. Minimizing $E_{\mathrm{dev}}$ with respect to $r_{\mathrm{c}}$ gives:

$$
r_{\mathbf{c}} \approx \frac{K}{\gamma_{0}}
$$


With our values of $K$, we find $r_{\mathrm{c}} \approx 150 \AA$ in the $\mathrm{C}_{12} \mathrm{EO}_{6}$ and $r_{\mathrm{c}} \approx 1300 \AA$ in C8HET. These estimates are in agreement with experiment. With the value of $K$ given by the measurements of the buckling instability ( $K_{\text {app }} \approx 0.1$ dyn [6]), we would find $r_{\mathrm{c}} \approx 2 \mathrm{~mm}$, which is impossible.

Let us now consider the second possibility, i.e., a core filled with isotropic liquid. In this case, the core energy reads:

$$
E_{\mathrm{c}} \approx 2 \pi r_{\mathrm{c}} \gamma_{0}+\pi r_{\mathrm{c}}^{2} \frac{L \Delta T}{T_{\mathrm{c}}}
$$

where the first term corresponds to the surface energy and the second one to the bulk energy of the undercooled liquid (with $\Delta T=T_{c}-T$ ). Minimization of the total energy (21) with respect to $r_{\mathrm{c}}$ gives:

$$
r_{\mathrm{c}} \approx \frac{T_{\mathrm{c}}}{2 L \Delta T}\left(\sqrt{\gamma_{0}^{2}+\frac{2 K L \Delta T}{T_{\mathrm{c}}}}-\gamma_{0}\right)
$$

With our values of $K$ we find at $\Delta T=1{ }^{\circ} \mathrm{C}, r_{\mathrm{c}} \approx 70 \AA$ in $\mathrm{C}_{12} \mathrm{EO}_{6}$ and $r_{\mathrm{c}} \approx 350 \AA$ in C8HET. As expected the core radii are smaller than in the previous model. In particular the value obtained for the discotic liquid crystal is too small and incompatible with observations. Finally, we calculate $r_{\mathrm{c}} \approx 6 \mu \mathrm{m}$ with $K_{\mathrm{app}}=0.1 \mathrm{dyn}$, which is also incompatible with experiment.

In conclusion, only the first model, assuming that the core is filled with perfect hexagonal mesophase, is compatible with observations. This calculation also suggests that our values of $K$ are usable whatever the scale of the deformation, as long as only static properties are considered.

This conclusion is reinforced by another observation. Indeed, we know from previous work [15] that discontinuity walls are unstable at long time (many days) with respect to the formation of walls of developable domains of macroscopic size when $\theta_{0} \geq 60^{\circ}$. This spontaneous transformation was observed both in lyotropic and thermotropic systems. It confirms that the curvature energy stored within developable domains is always very small, which is compatible with our current estimate of $K$.

In conclusion, our measurements again raise the question as to why dynamic measurements of buckling instability under rapid compression or dilation give much larger values of $K_{\text {app }}$. A possible answer proposed by Prost [8] could be that ends of columns linked together by screw dislocations make the system stiffer at "high" frequency ( $\mathrm{kHz}$ or more). These point defects could also be responsible for the "abnormal" Rayleigh scattering produced by these phases [29] (without the necessity to resort to some 3D-solid-like elasticity as proposed in Ref. [6a]). But there is so far no direct experimental evidence for these defects. Also this interpretation supposes that defects are frozen and do not move by permeation during the buckling process (which is quite possible). It would be thus very interesting in the future to measure $K_{\text {app }}$ as a function of frequency.

\section{Acknowledgments}

This work has been supported by DRET contract N 92/1313 and CNES contract N 94/0253. We thank A. Collet for lending us the calorimeter and A.M. Levelut, M. Clerc, L. Lejcek and Y. Galerne for very useful discussions. 


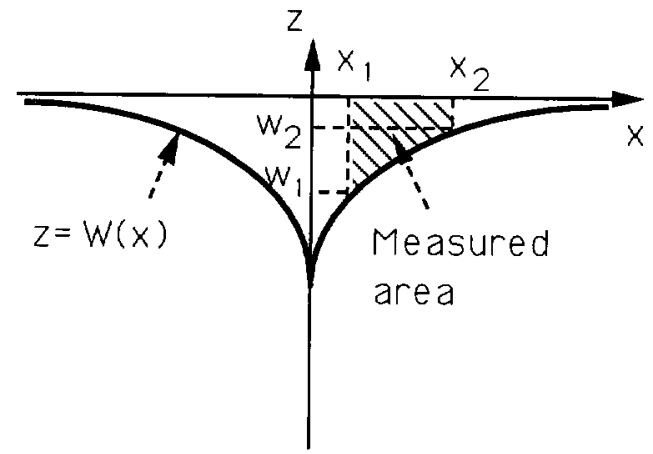

Fig. 13 - Definition of the measured quantities $x_{1}, w_{1}, \ldots$ in the method of Arbel and Cahn

\section{Appendix A}

\section{The Method of Arbel and Cahn for Measuring Surface Tension in Anisotropic Materials}

In 1977, Arbel and Cahn proposed a graphic method to measure the anisotropic surface tension of a solid-liquid interface from the shape of grain- boundary grooves which are observed in a temperature gradient [18]. Their method allows a direct determination of the vector function $\boldsymbol{\xi}=\gamma \mathbf{n}+\gamma^{\prime} \mathbf{t}$ in terms of distances and areas directly measurable on the micrographs. The vectors $\mathbf{n}$ and $\mathbf{t}$ are the unit vectors respectively normal and tangent to the interface of equation $z=w(x)$ (Fig. 13). Also $\gamma^{\prime}=\mathrm{d} \gamma / \mathrm{d} \phi$ where $\phi$ is the local angle between the normal to the interface and the striations (directly measurable on the micrographs). Of course, Arbel and Cahn do their calculations by assuming that the crystal is a monodomain on each side of the grain boundary. This assumption is not rigorously satisfied in our experiment because the columns are generally bent and centered on the core of a $S=1 / 2$ disclination. Let $h$ be the groove size and $D$ the distance between the groove and the center of curvature of the columns. This curvature effect introduces two corrections in the usual Gibbs-Thomson equation $\left(\gamma+\gamma^{\prime \prime} / R=L \Delta T / T_{\mathrm{c}}\right)$ : a geometrical one of the order of $\left(\gamma^{\prime \prime} / R\right)(h / D)$ and another due to the curvature elasticity of the order of $K / R^{2}$ [30]. The latter correction is always negligible because $R \gg K / \gamma \approx 0.01 \mu \mathrm{m}$. By contrast, the former can be important, except for the grooves for which $h / D<10 \%$. To simplify, we only measured these grooves for which we can directly apply the theory of Arbel and Cahn. Their results can be summed up in two equations:

$$
\begin{gathered}
\left(\xi_{x}\right)_{x=x_{1}}=\frac{G L}{T_{0}} \int_{x_{1}}^{x_{2}} w(x) \mathrm{d} x+\left(\xi_{x}\right)_{\boldsymbol{x}=x_{2}} \\
\left(\xi_{z}\right)_{x=x_{2}}=\frac{G L}{2 T_{0}}\left[w^{2}\left(x^{2}\right)-w^{2}\left(x_{1}\right)\right]+\left(\xi_{z}\right)_{x=x_{1}}
\end{gathered}
$$

In the first equation, the integral represents the hatched surface area in Figure 13. Because we know the $\gamma(\phi)$-dependence from the experimental Wulff plot, each of these two equations is in itself sufficient to determine $\gamma_{0}$. The values of $\gamma_{0}$ obtained separately from these two equations are in excellent agreement. 


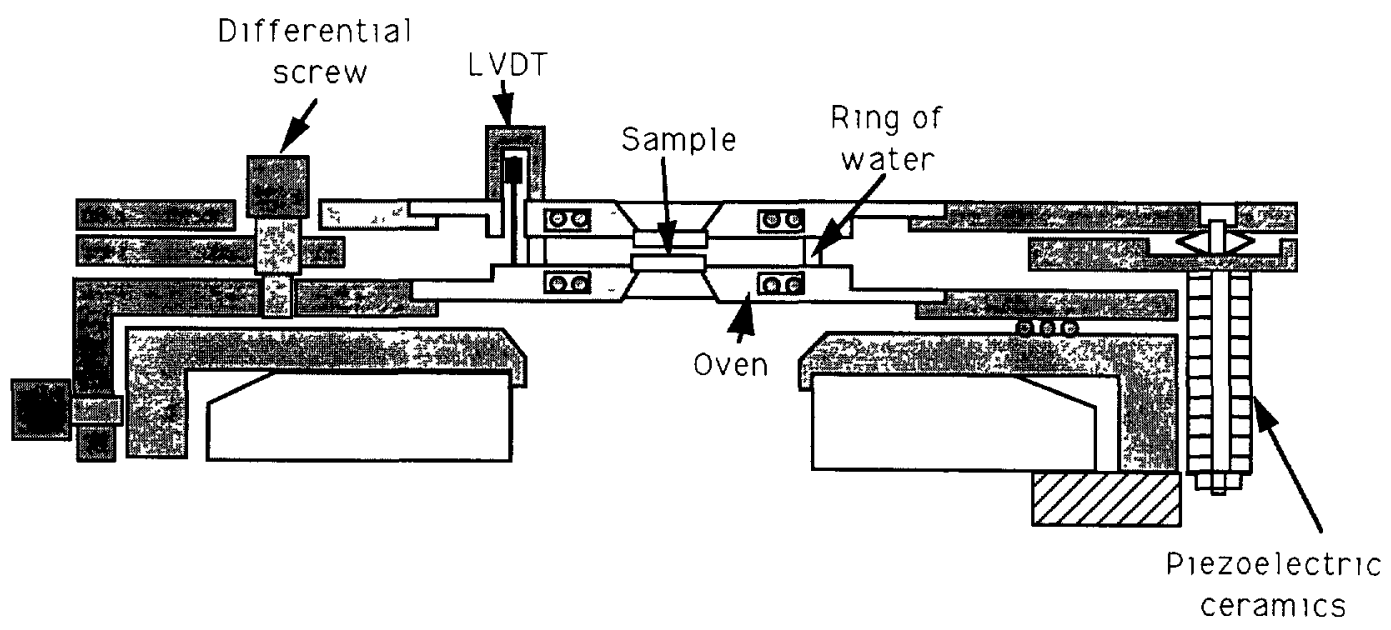

Fig. 14. - Schematic representation of the dilation cell used to measure the elastic modulus $B$.

\section{Appendix B}

\section{Young Modulus Measurement}

Our experimental set-up is described in detail in reference [22]. The sample is deformed by three stacks of piezoelectrics through a rigid frame (Fig. 14). It is prepared between two circular glass plates of diameter $1 \mathrm{~cm}$, polished to $\lambda / 10$. The parallelism (better than $2 \times 10^{-4} \mathrm{rd}$ ) and the spacing (usually between 25 and $400 \mu \mathrm{m}$ ) between the two plates is adjusted with three differential screws within $\pm 1 \mu \mathrm{m}$. A ring of water surrounds the sample to avoid evaporation. This precaution allows us to work during one hour without visible variations of the water concentration. After this time, the sample is changed because the sample starts to dry on the sides. A planar orientation of the columns (fan-shaped texture) is obtained by slowly freezing the sample from its isotropic phase. The temperature is stable to about $1 / 100{ }^{\circ} \mathrm{C}$ but is not perfectly homogeneous within the sample because of the presence of the observation windows and of the LVDT (linear variable differential transformer) which is fixed on the oven to measure the sample deformation. Indeed this sensor produces a small amount of heat, so that there exists a small temperature gradient across the sample of the order of $0.05^{\circ} \mathrm{C} / \mathrm{cm}$. Thus, it is impossible to approach the transition to better than $0.05^{\circ} \mathrm{C}$ which is of the same order of magnitude as the freezing range of our mixture.

In order to determine the mechanical response of the sample to a sinusoidal deformation, we measure with the LVDT (Schaevitz MHR005) and a lock-in amplifier (Stanford SR850) the displacement $a_{1}(t)$ and its phase shift with the ceramics displacement $u(t)$. We used a sinusoidal deformation of very small amplitude (less than $100 \AA$ at the level of the sample) and frequencies varying between 1 and $100 \mathrm{~Hz}$. We checked that all our results were independent of the amplitude chosen (linear regime). Also we found that inertial terms are negligible in this range of frequency and that the cell behaves elastically as a set of springs (Fig. 15). In this equivalent scheme (the simplest one we have found to explain all our experimental results), $a_{1}(t)$ is the displacement which is measured with the LVDT (slightly different from the sample thickness variation because of the finite tightness of the oven), $u(t)$ the ceramics displacement, 


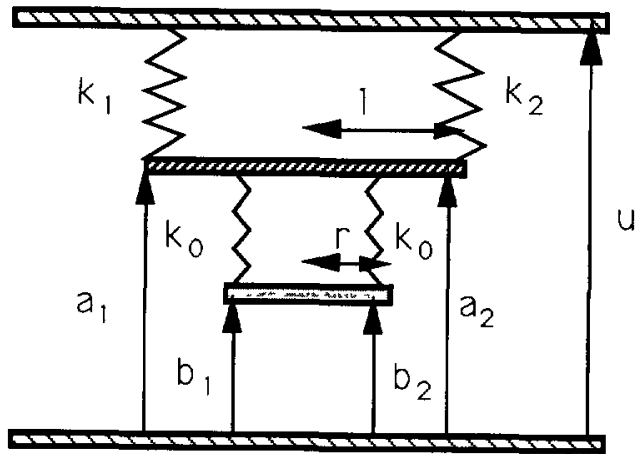

Fig. 15. - Equvalent mechanical model of the dilation cell.

$k_{1}$ and $k_{2}$ the force constants of the metal rods on which the oven is suspended and which are secured to the thick rigid metal ring which transmits the ceramic displacements. Finally, $k_{0}$ is the force constant of the oven itself, that we expect to be larger than $k_{1}$ and $k_{2}$, both of the same order of magnitude by construction Also $r$ is the glass plate radius and $l$ the distance between the center of the sample and the LVDT. Finally $b_{1}$ and $b_{2}$ are the displacements on both sides of the sample (slighly different because of the asymmetry of the cell).

Let us now see how to determine experimentally these three force constants $k_{\imath}(i=0,1,2)$. To do that experimentally, we used a silicone oil (Rhodorsil 47V1000 from Prolabo) of viscosity $\eta=10.2$ Poise, that we know to be Newtonian at low frequency (up to $10 \mathrm{kHz}$ ), and we measured the amplitude ratio $\left|a_{1}\right| /|u|$ as well as the phase shift $\varphi$ between $a_{1}$ and $u$ for a sinusoidal excitation:

$$
\begin{gathered}
u=|u| \exp (i \omega t) \\
a_{1}=\left|a_{1}\right| \exp (\imath \omega t+\imath \varphi)
\end{gathered}
$$

The equations of motion (for forces and torques) can be written for a viscous sample of thickness $d$ within the lubrication approximation [31] (which is valid here because of the extreme smallness of the displacements) in the form:

$$
\begin{gathered}
\beta_{1} k_{0}+\beta_{2} k_{0}+k_{1}-\alpha_{1}\left(k_{0}+k_{1}\right)+k_{2}-\alpha_{2}\left(k_{0}+k_{2}\right)=0 \\
k_{1} l-k_{2} l+\beta_{1} k_{0} r-\beta_{2} k_{0} r-\alpha_{1}\left(k_{1} l+\frac{k_{0} r_{2}}{l}+\alpha_{2}\left(k_{2} l+\frac{k_{0} r_{2}}{l}\right)=0\right. \\
\alpha_{1} k_{0}+\alpha_{2} k_{0}-\beta_{1}\left(k+\frac{3 I \eta r^{2}}{4 d^{3}}\right)-\beta_{2}\left(k+\frac{3 I \eta r^{2} \omega}{4 d^{3}}\right)=0 \\
-\frac{\alpha_{1} k_{0} r^{4}}{l}+\frac{\alpha_{2} k_{0} r^{4}}{l}-\beta_{2}\left(k r^{3}-\frac{I \eta r^{5} \omega}{16 d^{3}}\right)+\beta_{1}\left(k_{0} r^{3}+\frac{I \eta r^{5} \omega}{16 d^{3}}\right)=0
\end{gathered}
$$

with

$$
I=\mathrm{e}^{2 \pi / 2}, \quad \alpha_{1}=\frac{a_{\imath}}{u} \quad \text { and } \quad \beta_{\imath}=\frac{b_{\imath}}{u}
$$

This set of equations can be solved analytically using Mathematica, which gives:

$$
\alpha_{1}=\frac{a_{1}}{u}=\frac{N}{D}
$$



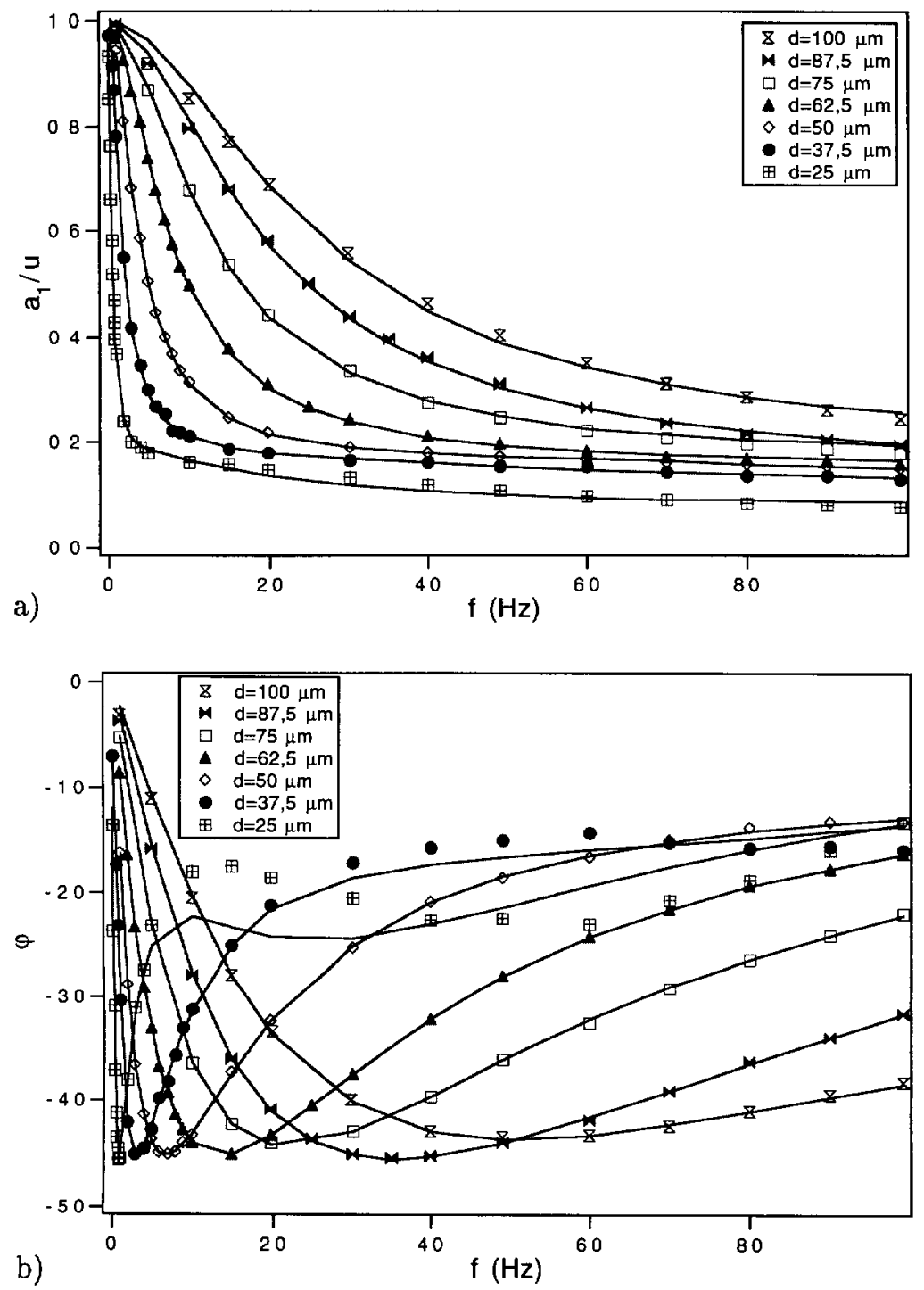

Fig. 16. - Calibration curves of the dilation cell obtained with the Newtonian silicone oil 47V1000. Points are experımental and curves are the best fits obtained from the model.

with

$$
\begin{gathered}
N=1+\omega\left(\frac{13 I}{16 \omega_{0}}-\frac{1703 I}{4608 \omega_{1}}+\frac{1753 I}{4608 \omega_{2}}\right)+\omega^{2}\left(-\frac{3}{64 \omega_{0}^{2}}+\frac{119}{6144 \omega_{0} \omega_{1}}-\frac{169}{6144 \omega_{0} \omega_{2}}\right) \\
D=1+\omega\left(\frac{13 I}{16 \omega_{0}}+\frac{1703 I}{4608 \omega_{1}}+\frac{1753 I}{4608 \omega_{2}}\right) \\
-\omega^{2}\left(\frac{3}{64 \omega_{2}}+\frac{169}{6144 \omega_{0} \omega_{1}}+\frac{169}{6144 \omega_{0} \omega_{2}}+\frac{25}{3072 \omega_{1} \omega_{2}}\right)
\end{gathered}
$$




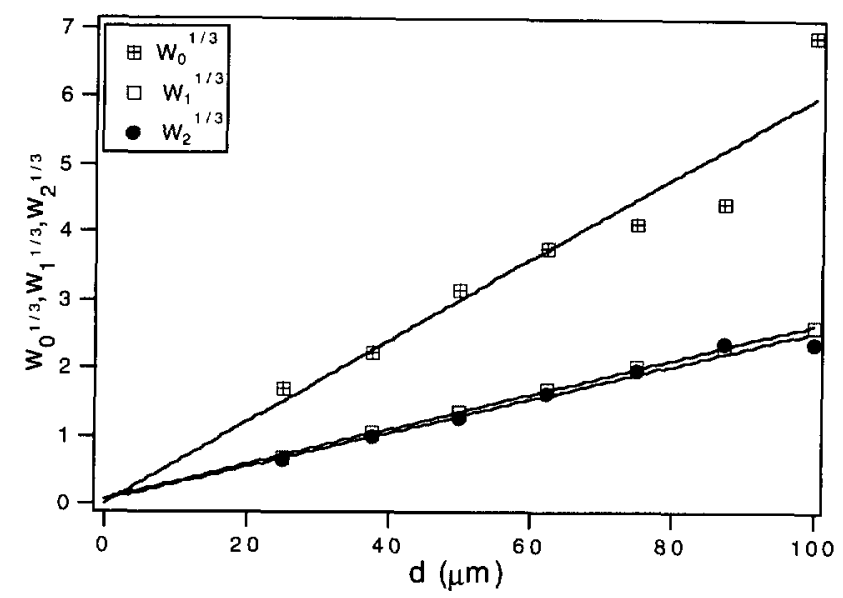

Fig. 17. - Parameters $\omega_{2}$ used in Figure 16 to fit the experimental data as a function of sample thickness $d$.

and

$$
w_{\imath}=\frac{d^{3} k_{\imath}}{\eta r^{2}}
$$

From this law it is possible to extract both the ratio $\left|a_{1}\right| /|u|$ and the phase shift $\varphi$ that we measure experimentally. These two quantities depend on frequency $(f=2 \pi / w)$ and on unknown parameters $\omega_{2}$.

In Figure 16 we plotted the experimental ratio $\left|a_{1}\right| /|u|$ and the phase shift $\varphi$ as a function of frequency for various values of the sample thickness as well as their best fit with the theoretical law (B.3.a). For each thickness, we determined a triplet of values $\left(\omega_{0}, \omega_{1}, \omega_{2}\right)$ that we reported as a function of the thickness $d$ in Figure 17. We see that each $\omega_{\imath}^{1 / 3}$ is proportional to $d$ as predicted (see Eq. (B.3b)). From the slopes of these three curves, we deduce the force constants in CGS units:

$$
\begin{aligned}
& k_{0}=3.77 \times 10^{9} \\
& k_{1}=2.53 \times 10^{8} \\
& k_{2}=2.03 \times 10^{8}
\end{aligned}
$$

As expected $k_{1}$ and $k_{2}$ are of the same order of magnitude. Nevertheless we see that there exists a non negligible asymmetry (of about $20 \%$ ) in the elastic rigidity of our system. This is easily understandable because the bend modulus $k_{0}$ of the rods which support the oven varies as the third power of their thickness (of the order of $1 \mathrm{~mm}$ ). That means that their thickness difference is of the order of $0.07 \mathrm{~mm}$, which is quite reasonable.

To test our calibration of the cell, we measured the compressibility modulus of the smectic A phase of the thermotropic liquid crystal 8OCB. We found for example $B=7.3 \times 10^{7} \mathrm{erg} / \mathrm{cm}^{3}$ at $f=100 \mathrm{~Hz}$ and $\Delta T=T-T_{\mathrm{NA}}=1^{\circ} \mathrm{C}$. This value is in good agreement with previous measurements [32].

We can now measure the elastic modulus $B$ of our mixture. More precisely, what we measure in this experiment is the compressibility modulus of the hexagonal array ( $B_{1}$ with the notation of Ref. [7]). In Figure 18, we plotted the ratio $\left|a_{1}\right| /|u|$ and the phase shift $\varphi$ as a function of time for a sample of thickness $d=400 \mu \mathrm{m}$ at frequency $f=90 \mathrm{~Hz}$. We see that about $20 \mathrm{mn}$ 

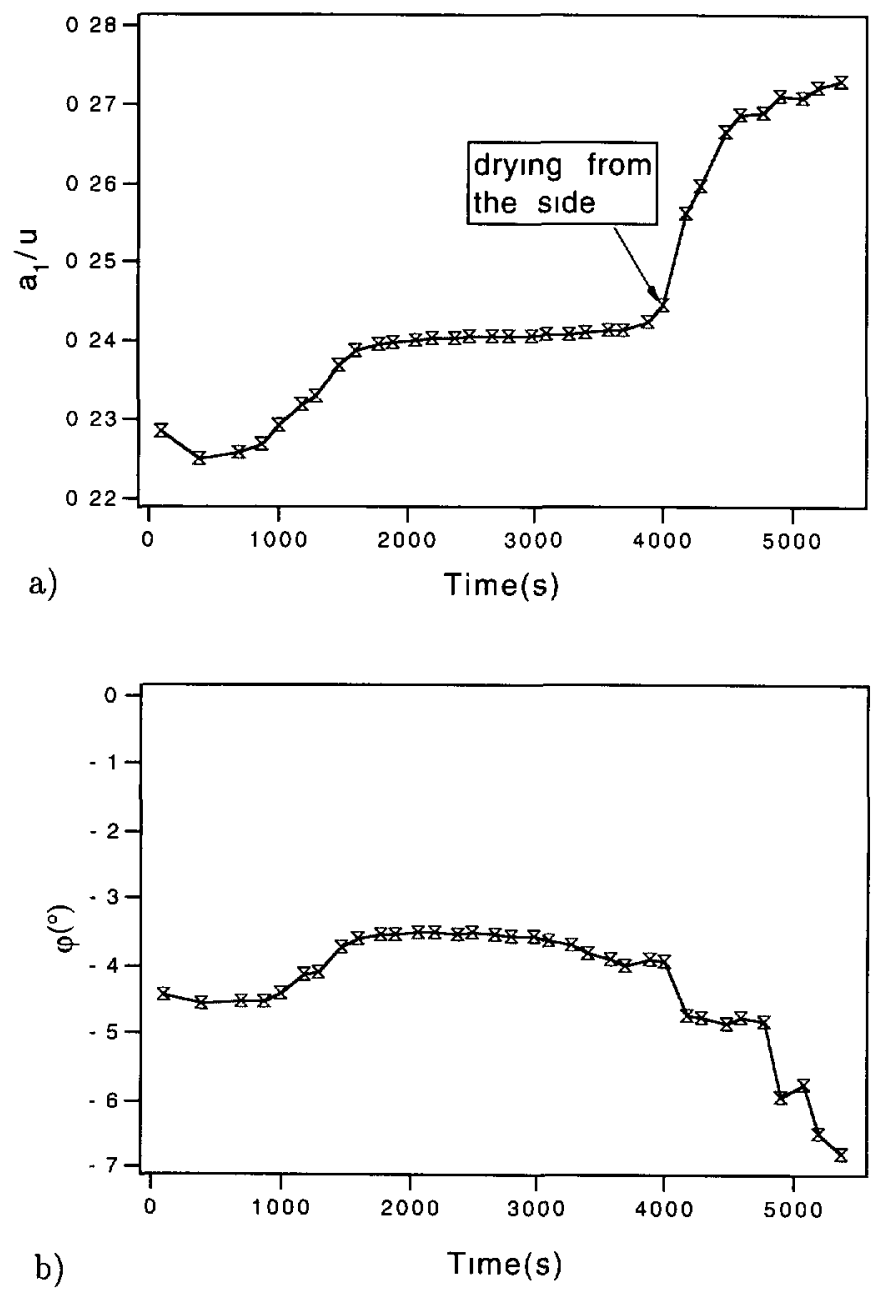

Fig. 18. - Time evolution of the amplitude (a) and the phase (b) $\left(\mathrm{C}_{12} \mathrm{EO}_{6}\right.$ at $\left.T=36.67{ }^{\circ} \mathrm{C}\right)$.

are necessary to obtain a stable signal after the sample has been cooled down from its isotropic liquid. On the other hand, we observe one hour later a brutal increase of the amplitude and a strong decrease of the phase shift: this accident clearly occurs when the sample starts to dry and to melt on the sides (see the phase diagram). On the plateau, the phase shift is also very small, which means that the response of the sample is essentially elastic at this frequency. In Figure 19, we plotted $\left|a_{1}\right| /|u|$ and $\varphi$ at saturation as a function of frequency $f$ for a sample of thickness $d=400 \mu \mathrm{m}$ (we have chosen this thickness because it is a good compromise between obtaining a good ratio signal/noise and keeping a good planar orientation). We see that the response is elastic above $20 \mathrm{~Hz}$ (within 99\%) while plastic effects occur below. Also the amplitude ratio saturates above $80 \mathrm{~Hz}$. For this reason we measured $B$ at $f=90 \mathrm{~Hz}$. As before, we must solve the motion equations for calculating $B$. If one assumes that dissipation is negligible, i.e. the sample is elastic, of Young modulus $B$, the equations to solve read:

$$
\beta_{1} k_{0}+\beta_{2} k_{0}-\alpha_{1}\left(k_{0}+k_{1}\right)+k_{1}-\alpha_{2}\left(k_{0}+k_{2}\right)+k_{2}=0
$$




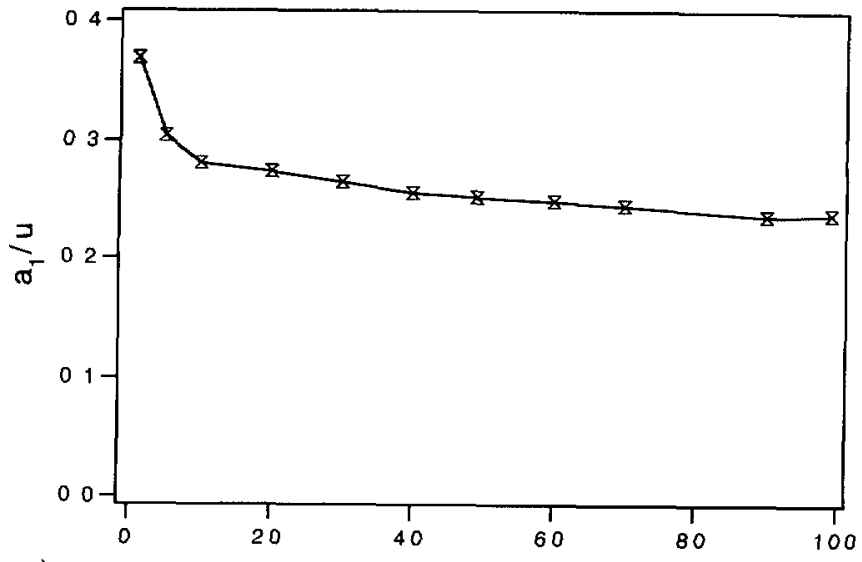

a)

$f(\mathrm{~Hz})$

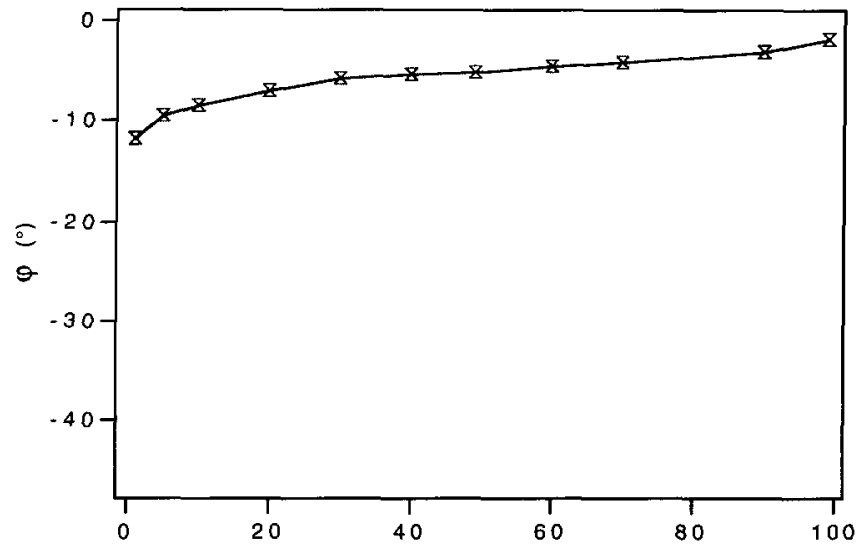

b) $\quad f(H z)$

Fig. 19 - Amplitude (a) and phase (b) as a function of frequency $\left(\mathrm{C}_{12} \mathrm{Eo}_{6}\right.$ at $\left.T=36.67^{\circ} \mathrm{C}\right)$.

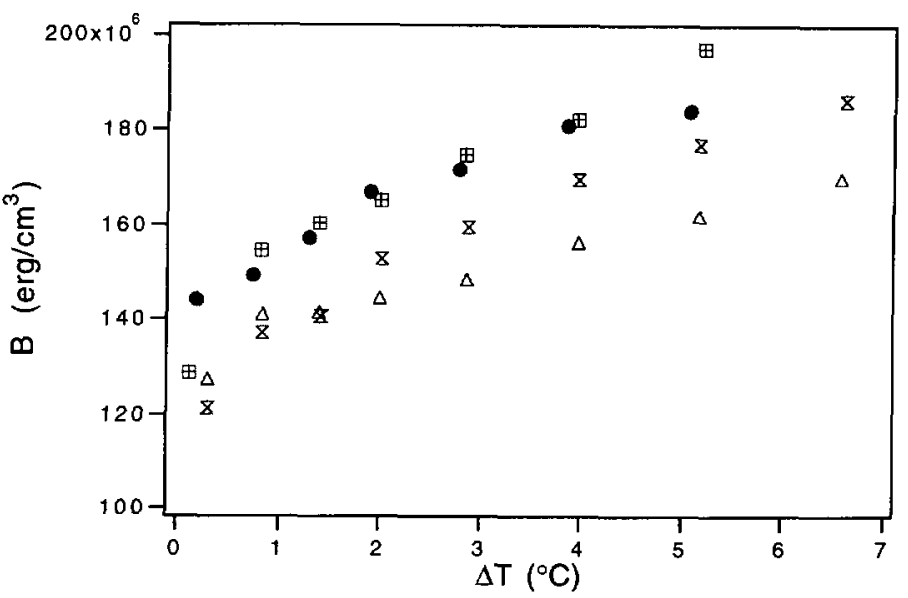

Fig. 20. - Elastic modulus found at $f=90 \mathrm{~Hz}$ as a function of temperature shift $\Delta T=T_{\text {transition }}-T$ $\left(\mathrm{C}_{12} \mathrm{EO}_{6}\right)$. 


$$
\begin{gathered}
k_{1} l-k_{2} l+\beta_{1} k r-\beta_{2} k_{0} r-\alpha_{1}\left(k_{1} l+\frac{k_{0} r^{2}}{l}\right)+\alpha_{2}\left(k_{2} l+\frac{k_{0} r^{2}}{l}\right)=0 \\
\alpha_{1} k_{0}+\alpha_{2} k_{0}-\beta_{1}\left(\frac{B}{2 d}+k_{0}\right)-\beta_{2}\left(\frac{B}{2 b}+k_{0}\right)=0 \\
-\frac{\alpha_{1} k_{0} r^{2}}{l}+\frac{\alpha_{2} k_{0} r^{2}}{l}-\beta_{2}\left(k_{0} r+X\right)+\beta_{1}\left(k_{o} r+X\right)=0
\end{gathered}
$$

with

$$
X=\frac{B S}{8 \pi r d}, \quad S=\pi r^{2}
$$

The resolution of this set of equations gives, using experimental values (B.4a-c):

$$
\alpha_{1}=\frac{3.36 \times 10^{20}+1.7 \times 10^{11} B^{*}+8.50 B^{*} 2}{3.36 \times 10^{20}+8.34 \times 10^{11} B^{*}+1.02 \times 10^{2} B^{* 2}}(\text { CGS units })
$$

where $B^{*}=B / d$.

As expected $\alpha_{1}$ is a real number and $\varphi=0$ (elastic behavior). This equation gives the elastic modulus $B$ as a function of the amplitude ratio $\alpha_{1}$ which is measured experimentally:

$$
B^{*}=\frac{B}{d}=6.06 \times 10^{10} \frac{0.20-\alpha_{1}+0.90 \sqrt{0.03-0.24 \alpha_{1}+\alpha_{1}^{2}}}{14.7 \alpha_{1}-1.23}
$$

Our results as a function of temperature are given in Figure 20 for three different samples. We see that $B$ decreases when the temperature increases and tends to a finite value at the transition temperature of the order of:

$$
B_{\text {transition }} \approx 1.2 \pm 0.1 \times 10^{8} \mathrm{erg} / \mathrm{cm}^{3} \text {. }
$$

At low frequency $\left(f<20 \mathrm{~Hz}\right.$ ), the situation is more complicated, the amplitude ratio $\left|\alpha_{1}\right|$ increasing while $\varphi$ decreases. Nevertheless, $\varphi$ always remains very small $\left(\varphi \approx-10^{\circ}\right.$ at $f=$ $1 \mathrm{~Hz}$ ) which means that the sample response remains essentially elastic. The variation of the amplitude ratio is thus mainly due to a decrease of the elastic modulus. For instance, $B(1 \mathrm{~Hz}) \approx 5 \times 10^{7} \mathrm{erg} / \mathrm{cm}^{3}$ at $\Delta T=1{ }^{\circ} \mathrm{C}$ which is a value about three times smaller than that found at high frequency at the same temperature. This variation of $B$ with frequency could be due either to pinned dislocations or to undulations of the columns which systematically develop in samples when they are cooled down (striations).

\section{References}

[1] Ekwall P., Advances in Liquid Crystals, Vol. 1, G.H. Brown, Ed. (Academic Press, New York, 1975) p. 1.

[2] Mitchell D.J., Tiddy G.J.T , Waring L., Bostock T. and McDonald M.P., J. Chem. Soc., Faraday Trans. I 79 (1983) 975.

[3] Clerc M., Levelut A.M. and Sadoc J.F., J. Phys. II France 1 (1991) 1263.

[4] Cagnon M., Gharbia M. and Durand G., Phys. Rev. Lett. 53 (1984) 938.

[5] Gharbia M., Cagnon M. and Durand G., J. Phys Lett 46 (1985) L-683.

[6] a) Gharbia M., Othman T., Gharbi A., Destrade C. and Durand G., Phys. Rev. Lett. 68 (1992) 2031; b) Brand H.R. and Pleiner H., Phys. Rev. Lett. 69 (1992) 987; c) Durand G., Phys. Rev Lett. 69 (1992) 987. 
[7] Kléman M. and Oswald P., J. Phys. France 43 (1982) 655.

[8] Prost J., Lıq. Cryst 8 (1990) 123.

[9] Oswald P., Moulin M., Metz P., Géminard J.C., Sotta P. and Sallen L., J. Phys. III France 3 (1993) 1891.

[10] Bouligand Y., J. Phys. France 41 (1980) 1297; Kléman M., J. Phys. France 41 (1980) 737.

[11] Gémınard J.C., Thèse, Unıversité Claude Bernard - Lyon I (1993).

[12] We shall show in a forthcoming article that meniscus effects are negligible, as long as the contact $\alpha$ between the interface and the glass plates is close to $90^{\circ}$. In our system, $\alpha \approx 70^{\circ}$ and the error in determining the Wulff plot is of the order of $10 \%$.

[13] Wulff G Z., Kristallogr. Mineral. 34 (1901) 449.

[14] Woodruff D.P, The Solid-Liquid Interface (Cambridge University Press, 1973).

[15] Oswald P. and Kléman M., J. Phys. France 42 (1981) 1461; Oswald P., Thèse de 3ème Cycle, Orsay (1981).

[16] Nozières Ph., Solids Far From Equilibruum, C. Godrèche, Ed. (Cambrıdge University Press, 1992).

[17] Clunie J.S., Goodman J.F. and Symons P.C., Trans. Faraday Soc. 65 (1969) 287.

[18] Arbel E. and Cahn J.W., Surf. Scr. 66 (1977) 14.

[19] Williams C., Thèse d'Etat, Série A, No. d'ordre 1646, Orsay (1976).

[20] Chandrasekhar S., Liquid Crystals (Cambridge University Press, 1992).

[21] Kléman M., Points - Lignes - Parois,(Les Editions de Physique, Les Ulis, 1977).

[22] Oswald P. and Le Fur, $C$ R. Acad. Scı (Paris) Ser. B 297 (1983) 699.

[23] This value is much less precise than in lyotropics because of problems of contact angle and hysteresis on the surfaces. Also the contact angle is close to $60^{\circ}$ (see Géminard J.C, Thèse à Unıersité de Lyon I, 1993) and meniscus effects should change the Wulff plot significantly. Our value of $\gamma_{2}$ is thus only indicative.

[24] Oswald P, J. Phys. France 49 (1988) 1083.

[25] Raghunathan V.A., Madhusudana N.V. and Chandrasekhar S., Mol. Cryst. Lıq. Cryst. 148 (1987) 77.

[26] Davıdson P., Clerc M., Ghosh S S , Maliszewskyj N.C., Heiney P.A., Hynes J. and Smith A.B, preprint

[27] Fournier J.B. and Durand G., J. Phys. II France 1 (1991) 845.

[28] Oswald P., J. Phys. Lett. France 42 (1990) 895.

[29] Galerne Y., private communication.

[30] Valance A., Contribution a la théorie dynamique des fronts de croissance des cristaux nématiques et des eutectiques lamellaires, Thèse de Doctorat, Université de Paris 7 (1993).

[31] Batchelor G.K., Fluid Dynamics (Cambridge University Press, 1981), p. 219.

[32] Ricard L. and Prost J., J. Phys. France 42 (1981) 861. 